Turk. J. Math. Comput. Sci.

13(1)(2021) 221-225

(C) MatDer

DOI : $10.47000 /$ tjmcs. 884526

\title{
Fixed Point Results for Zamfirescu Mappings in $A$-metric Spaces
}

\author{
IsA YILDIRIM(iD) \\ Department of Mathematics, Faculty of Science, Ataturk University, 25240 Erzurum, Turkey.
}

Received: 21-02-2021 • Accepted: 23-06-2021

Aвstract. In the present paper, we extend the Zamfirescu results ( [9]) to $A$-metric spaces. Firstly, we define the notion of Zamfirescu mapping in $A$-metric spaces. After, we also obtain a fixed point theorem for such mappings. The established results carry some well-known results from the literature (see $[2,3,5,9])$ to $A$-metric spaces. An appropriate example is also given.

2010 AMS Classification: 47H09, 47H10, 54H25

Keywords: $A$-metric spaces, Zamfirescu mappings, fixed point.

\section{Introduction and Preliminaries}

Zamfirescu's fixed point theorem is one of the most important extensions of Banach contraction principle. In 1972, Zamfirescu [9] obtained the following a very interesting fixed point theorem.

Theorem 1.1. Let $(U, d)$ be a complete metric space and let $T: U \rightarrow U$ be a mapping for which there exists the real numbers $a, b$ and $c$ satisfying $a \in(0,1) ; b, c \in\left(0, \frac{1}{2}\right)$ such that for each pair $u, v \in U$, at least one of the following conditions holds:

$\left(Z_{1}\right) d(T u, T v) \leq a d(u, v)$

$\left(Z_{2}\right) d(T u, T v) \leq b[d(u, T u)+d(v, T v]$

$\left(Z_{3}\right) d(T u, T v) \leq c[d(u, T v)+d(v, T u]$.

Then $T$ has a unique fixed point $u^{*}$ and the Picard iteration $\left\{u_{n}\right\}$ defined by $u_{n+1}=T u_{n}$ converges to $u^{*}$ for any arbitrary fixed $u_{0} \in U$.

An operator $T$ satisfying the contractive conditions $\left(Z_{1}\right),\left(Z_{2}\right)$ and $\left(Z_{3}\right)$ in the above theorem is called Zamfirescu mapping. Zamfirescu's theorem is a generalization of Banach's, Kannan's and Chatterjea's fixed point theorems (see $[2,3,5])$. Many researchers studied to obtain new classes of contraction mappings in different metric spaces. Some of them are $D^{*}$-metric space (see [8]), $G$-metric space (see [6]), $S$-metric space (see [7]), $A$-metric space (see [1]), etc., as a generalization of the usual metric space. These generalizations helped the development of fixed point theory.

In 2006, Mustafa and Sims [6] introduced the notion of $G$-metric space. After, Sedghi et al. [7] defined the concept of $D^{*}$-metric and $S$-metric spaces. Also, they proved some fixed point theorems in such spaces. Every $G$-metric space is a $D^{*}$-metric space and every $D^{*}$-metric space is an $S$-metric space. That is, $S$-metric space is a generalization of the $G$-metric space and the $D^{*}$-metric space.

In 2015, Abbas et al. [1] introduced the concept of an A-metric space as follows:

Email address: isayildirim@atauni.edu.tr (I. Yildirim) 
Definition 1.2. Let $U$ be nonempty set. Suppose a mapping $A: U^{t} \rightarrow \mathbb{R}$ satisfies the following conditions:

$\left(A_{1}\right) A\left(u_{1}, u_{2}, \ldots, u_{t-1}, u_{t}\right) \geq 0$,

$\left(A_{2}\right) A\left(u_{1}, u_{2}, \ldots, u_{t-1}, u_{t}\right)=0$ if and only if $u_{1}=u_{2}=\ldots=u_{t-1}=u_{t}$,

$\left(A_{3}\right) A\left(u_{1}, u_{2}, \ldots, u_{t-1}, u_{t}\right) \leq A\left(u_{1}, u_{1}, \ldots,\left(u_{1}\right)_{t-1}, v\right)+A\left(u_{2}, u_{2}, \ldots,\left(u_{2}\right)_{t-1}, v\right)+\ldots+A\left(u_{t-1}, u_{t-1}, \ldots,\left(u_{t-1}\right)_{t-1}, v\right)+A\left(u_{t}, u_{t}, \ldots,\left(u_{t}\right)_{t-1}, v\right)$, for any $u_{i}, v \in U,(i=1,2, \ldots, t)$. Then, $(U, A)$ is said to be an $A$-metric space.

It is clear that the an $A$-metric space for $t=2$ reduces to ordinary metric $d$ and an $A$-metric space for $t=3$ reduces to $S$-metric spaces. So, an $A$-metric space is a generalization of the $G$-metric space, the $D^{*}$-metric space and the $S$-metric space.

Example 1.3 ([1]). Let $U=\mathbb{R}$. Define a function $A: U^{t} \rightarrow \mathbb{R}$ by

$$
\begin{aligned}
A\left(u_{1}, u_{2}, \ldots, u_{t-1}, u_{t}\right)= & \left|u_{1}-u_{2}\right|+\left|u_{1}-u_{3}\right|+\ldots+\left|u_{1}-u_{t}\right| \\
& +\left|u_{2}-u_{3}\right|+\left|u_{2}-u_{4}\right|+\ldots+\left|u_{2}-u_{t}\right| \\
& \vdots \\
& +\left|u_{t-2}-u_{t-1}\right|+\left|u_{t-2}-u_{t}\right|+\left|u_{t-1}-u_{t}\right| \\
= & \sum_{i=1}^{t} \sum_{i<j}\left|u_{i}-u_{j}\right| .
\end{aligned}
$$

Then $(U, A)$ is an $A$-metric space.

In 2017, Fernandez et al. [4] introduced the generalized Lipschitz mapping, Chatterjea's and Kannan's mappings in an $A$-cone metric space over Banach algebra. Also, they proved some fixed point theorems for the above mappings in complete $A$-cone metric space $(U, A)$ over Banach algebra.

Next, we state the following useful lemmas and definition.

Lemma 1.4 ( [1]). Let $(U, A)$ be an A-metric space. Then $A(u, u, \ldots, u, v)=A(v, v, \ldots, v, u)$ for all $u, v \in U$.

Lemma 1.5 ( [1]). Let $(U, A)$ be an A-metric space. Then for all $u, v, z \in U$ we have $A(u, u, \ldots, u, z) \leq(t-1) A(u, u, \ldots, u, v)+$ $A(z, z, \ldots, z, v)$ and $A(u, u, \ldots, u, z) \leq(t-1) A(u, u, \ldots, u, v)+A(v, v, \ldots, v, z)$.

Definition 1.6 ( $[1])$. Let $(U, A)$ be an $A$-metric space.

(i) A sequence $\left\{u_{n}\right\}$ in $U$ is said to converge to a point $u \in U$ if $A\left(u_{n}, u_{n}, \ldots, u_{n}, u\right) \rightarrow 0$ as $n \rightarrow \infty$,

(ii) A sequence $\left\{u_{n}\right\}$ in $U$ is called a Cauchy sequence if $A\left(u_{n}, u_{n}, \ldots, u_{n}, u_{m}\right) \rightarrow 0$ as $n, m \rightarrow \infty$,

(iii) The $A$-metric space $(U, A)$ is said to be complete if every Cauchy sequence in $U$ is convergent.

\section{Main Results}

In this section, following the ideas of Zamfirescu [9] we first introduce the notion of Zamfirescu mappings in $A$-metric space as follows:

Definition 2.1. Let $(U, A)$ be an $A$-metric space and let $T: U \rightarrow U$ be a mapping. $T$ is called a $A$-Zamfirescu mapping ( $A Z$ mapping), if and only if, there are real numbers, $0 \leq a<1,0 \leq b, c<\frac{1}{t}$ such that for all $u, v \in U$, at least one of the next conditions is true:

$$
\begin{gathered}
\left(A Z_{1}\right) A(T u, T u, \ldots, T u, T v) \leq a A(u, u, \ldots, u, v), \\
\left(A Z_{2}\right) A(T u, T u, \ldots, T u, T v) \leq b[A(T u, T u, \ldots, T u, u)+A(T v, T v, \ldots, T v, v)], \\
\left(A Z_{3}\right) A(T u, T u, \ldots, T u, T v) \leq c[A(T u, T u, \ldots, T u, v)+A(T v, T v, \ldots, T v, u)] .
\end{gathered}
$$

It is clear that if we take $t=2$ in the Definition 2.1, we obtain the definition of Zamfirescu [9] in ordinary metric space. Before giving the our main result in $A$-metric space, we need the following significant lemma.

Lemma 2.2. Let $(U, A)$ be an A-metric space and let $T: U \rightarrow U$ be a mapping. If $T$ is a AZ mapping, then there is $0 \leq \delta<1$ such that

$$
A(T u, T u, \ldots, T u, T v) \leq \delta A(u, u, \ldots, u, v)+t \delta A(T u, T u, \ldots, T u, u)
$$

and

for all $u, v \in U$.

$$
A(T u, T u, \ldots, T u, T v) \leq \delta A(u, u, \ldots, u, v)+t \delta A(T v, T v, \ldots, T v, u)
$$


Proof. Let's assume that $\left(A Z_{2}\right)$ is hold. From Lemma 1.5, we have

$$
\begin{aligned}
A(T u, T u, \ldots, T u, T v) \leq & b[A(T u, T u, \ldots, T u, u)+A(T v, T v, \ldots, T v, v)] \\
\leq & b\left[\begin{array}{r}
A(T u, T u, \ldots, T u, u)+(t-1) A(T v, T v, \ldots, T v, T u)] \\
+A(T u, T u, \ldots, T u, v)
\end{array}\right] \\
\leq & b[A(T u, T u, \ldots, T u, u)+(t-1) A(T v, T v, \ldots, T v, T u) \\
& +(t-1) A(T u, T u, \ldots, T u, u)+A(u, u, \ldots, u, v)] .
\end{aligned}
$$

Thus,

$$
(1-b(t-1)) A(T u, T u, \ldots, T u, T v) \leq t b A(T u, T u, \ldots, T u, u)+b A(u, u, \ldots, u, v) .
$$

From the fact that $0 \leq b<\frac{1}{t}$, we get

$$
A(T u, T u, \ldots, T u, T v) \leq \frac{b}{1-b(t-1)} A(u, u, \ldots, u, v)+\frac{t b}{1-b(t-1)} A(T u, T u, \ldots, T u, u) .
$$

Assume that $\left(A Z_{3}\right)$ is hold. From Lemmas 1.4 and 1.5, similarly we get

$$
\begin{aligned}
A(T u, T u, \ldots, T u, T v) \leq & c[A(T u, T u, \ldots, T u, v)+A(T v, T v, \ldots, T v, u)] \\
\leq & c\left[\begin{array}{r}
A(T u, T u, \ldots, T u, v)+(t-1) A(T v, T v, \ldots, T v, T u)] \\
+A(T u, T u, \ldots, T u, u)
\end{array}\right] \\
\leq & c[A(T u, T u, \ldots, T u, v)+(t-1) A(T v, T v, \ldots, T v, T u) \\
& +(t-1) A(T u, T u, \ldots, T u, v)+A(v, v, \ldots, v, u)] .
\end{aligned}
$$

Thus,

$$
(1-c(t-1)) A(T u, T u, \ldots, T u, T v) \leq t c A(T u, T u, \ldots, T u, v)+c A(u, u, \ldots, u, v) .
$$

From the fact that $0 \leq c<\frac{1}{t}$, we get

$$
A(T u, T u, \ldots, T u, T v) \leq \frac{c}{1-c(t-1)} A(u, u, \ldots, u, v)+\frac{t c}{1-c(t-1)} A(T u, T u, \ldots, T u, v) .
$$

Therefore, denoting by

$$
\delta=\max \left\{a, \frac{b}{1-b(t-1)}, \frac{c}{1-c(t-1)}\right\},
$$

we have that $0 \leq \delta<1$. Thus, the following inequalities hold:

$$
A(T u, T u, \ldots, T u, T v) \leq \delta A(u, u, \ldots, u, v)+t \delta A(T u, T u, \ldots, T u, u)
$$

and

for all $u, v \in U$.

$$
A(T u, T u, \ldots, T u, T v) \leq \delta A(u, u, \ldots, u, v)+t \delta A(T v, T v, \ldots, T v, u)
$$

Theorem 2.3. Let $(U, A)$ be a complete A-metric space and let $T: U \rightarrow U$ be an AZ mapping. Then $U$ has a unique fixed point and Picard iteration process $\left\{u_{n}\right\}$ defined by $u_{n+1}=T u_{n}$ converges to a fixed point of $T$.

Proof. Let $u_{0} \in U$ be arbitrary and $\left\{u_{n}\right\}$ be the Picard iteration as $u_{n+1}=T u_{n}$.

If we take $u=u_{n}$ and $v=u_{n-1}$ at the inequality (2.2), we obtain that

$$
\begin{aligned}
A\left(u_{n+1}, u_{n+1}, \ldots, u_{n+1}, u_{n}\right) & =A\left(T u_{n}, T u_{n}, \ldots, T u_{n}, T u_{n-1}\right) \\
& \leq \delta A\left(u_{n}, u_{n}, \ldots, u_{n}, u_{n-1}\right)+t \delta A\left(u_{n}, u_{n}, \ldots, u_{n}, u_{n}\right) .
\end{aligned}
$$

From above the inequality, we get

$$
\begin{aligned}
A\left(u_{n+1}, u_{n+1}, \ldots, u_{n+1}, u_{n}\right) \leq & \delta A\left(u_{n}, u_{n}, \ldots, u_{n}, u_{n-1}\right) \\
\leq & \delta^{2} A\left(u_{n-1}, u_{n-1}, \ldots, u_{n-1}, u_{n-2}\right) \\
& \vdots \\
\leq & \delta^{n} A\left(u_{1}, u_{1}, \ldots, u_{1}, u_{0}\right) \\
= & \delta^{n} A\left(u_{0}, u_{0}, \ldots, u_{0}, u_{1}\right) .
\end{aligned}
$$


Let $m>n$. Using Lemma 1.5 and the above inequality, we get

$$
\begin{aligned}
A\left(u_{n}, u_{n}, \ldots, u_{n}, u_{m}\right) \leq & (t-1) \delta A\left(u_{n}, u_{n}, \ldots, u_{n}, u_{n+1}\right)+A\left(u_{n+1}, u_{n+1}, \ldots, u_{n+1}, u_{m}\right) \\
\leq & (t-1) \delta A\left(u_{n}, u_{n}, \ldots, u_{n}, u_{n+1}\right)+(t-1) A\left(u_{n+1}, u_{n+1}, \ldots, u_{n+1}, u_{n+2}\right) \\
& +A\left(u_{n+2}, u_{n+2}, \ldots, u_{n+2}, u_{m}\right) \\
\leq & (t-1) \delta A\left(u_{n}, u_{n}, \ldots, u_{n}, u_{n+1}\right)+(t-1) A\left(u_{n+1}, u_{n+1}, \ldots, u_{n+1}, u_{n+2}\right) \\
& +\cdots+(t-1) A\left(u_{m-2}, u_{m-2}, \ldots, u_{m-2}, u_{m-1}\right) \\
& +A\left(u_{m-1}, u_{m-1}, \ldots, u_{m-1}, u_{m}\right) \\
= & (t-1)\left[\delta^{n} A\left(u_{0}, u_{0}, \ldots, u_{0}, u_{1}\right)+\delta^{n+1} A\left(u_{0}, u_{0}, \ldots, u_{0}, u_{1}\right)\right. \\
& \left.+\cdots+\delta^{m-2} A\left(u_{0}, u_{0}, \ldots, u_{0}, u_{1}\right)\right]+\delta^{m-1} A\left(u_{0}, u_{0}, \ldots, u_{0}, u_{1}\right) \\
= & (t-1) \delta^{n} A\left(u_{0}, u_{0}, \ldots, u_{0}, u_{1}\right)\left[1+\delta+\delta^{2}+\cdots+\delta^{m-n-2}\right] \\
& +\delta^{m-1} A\left(u_{0}, u_{0}, \ldots, u_{0}, u_{1}\right) \\
\leq & (t-1) \delta^{n} A\left(u_{0}, u_{0}, \ldots, u_{0}, u_{1}\right) \frac{\delta^{m}}{1-\delta}+\delta^{m-1} A\left(u_{0}, u_{0}, \ldots, u_{0}, u_{1}\right) \\
= & {\left[(t-1) \frac{\delta^{m+n}}{1-\delta}+\delta^{m-1}\right] A\left(u_{0}, u_{0}, \ldots, u_{0}, u_{1}\right) . }
\end{aligned}
$$

We know that $0 \leq \delta<1$ from Lemma 2.2. Suppose that $A\left(u_{0}, u_{0}, \ldots, u_{0}, u_{1}\right)>0$. If we take limit as $m, n \rightarrow \infty$ in above inequality we get

$$
\lim _{n, m \rightarrow \infty} A\left(u_{n}, u_{n}, \ldots, u_{n}, u_{m}\right)=0 .
$$

Therefore $\left\{u_{n}\right\}$ is a Cauchy sequence in $U$. Also, assume that $A\left(u_{0}, u_{0}, \ldots, u_{0}, u_{1}\right)=0$, then $A\left(u_{n}, u_{n}, \ldots, u_{n}, u_{m}\right)=0$ for all $m>n$ and $\left\{u_{n}\right\}$ is a Cauchy sequence in $U$. Since $(U, A)$ is a complete metric space, $u_{n} \rightarrow u^{*} \in U$ as $n \rightarrow \infty$.

We show that $u^{*}$ is a fixed point of $T$. From (2.1), we have

$$
\begin{aligned}
A\left(T u^{*}, T u^{*}, \ldots, T u^{*}, u^{*}\right) \leq & (t-1) A\left(T u^{*}, T u^{*}, \ldots, T u^{*}, T u_{n}\right)+A\left(T u_{n}, T u_{n}, \ldots, T u_{n}, u^{*}\right) \\
\leq & (t-1)\left[\delta A\left(u^{*}, u^{*}, \ldots, u^{*}, u_{n}\right)+t \delta A\left(u^{*}, u^{*}, \ldots, u^{*}, T u_{n}\right)\right] \\
& +A\left(u_{n+1}, u_{n+1}, \ldots, u_{n+1}, u^{*}\right) \\
= & (t-1)\left[\delta A\left(u^{*}, u^{*}, \ldots, u^{*}, u_{n}\right)+t \delta A\left(u^{*}, u^{*}, \ldots, u^{*}, u_{n+1}\right)\right] \\
& +A\left(u_{n+1}, u_{n+1}, \ldots, u_{n+1}, u^{*}\right) .
\end{aligned}
$$

If we take limit for $n \rightarrow \infty$ in above inequality, we obtain that $T u^{*}=u^{*}$. That is, $u^{*}$ is a fixed point of the mapping $T$. Now, we show that the uniqueness of fixed point of $T$. Assume that $u^{*}$ and $v^{*}$ are fixed point of $T$. That is, $T u^{*}=u^{*}$ and $T v^{*}=v^{*}$. From (2.1), we have

$$
\begin{aligned}
A\left(u^{*}, u^{*}, \ldots, u^{*}, v^{*}\right) & =A\left(T u^{*}, T u^{*}, \ldots, T u^{*}, T v^{*}\right) \\
& \leq \delta A\left(u^{*}, u^{*}, \ldots, u^{*}, v^{*}\right)+t \delta A\left(T u^{*}, T u^{*}, \ldots, T u^{*}, u^{*}\right) .
\end{aligned}
$$

Thus,

$$
A\left(u^{*}, u^{*}, \ldots, u^{*}, v^{*}\right) \leq \delta A\left(u^{*}, u^{*}, \ldots, u^{*}, v^{*}\right) .
$$

This implies that $A\left(u^{*}, u^{*}, \ldots, u^{*}, v^{*}\right)=0 \Longrightarrow u^{*}=v^{*}$ and hence, $T$ has a unique fixed point in $U$.

Remark 2.4. Putting $t=2$ in Theorem 2.3, we obtain the Theorem 1.1. Hence, Theorem 2.3 is a generalization of Theorem 1.1 of Zamfirescu [9] in A-metric space.

Example 2.5. Let $U=\mathbb{R}$. Define a function $A: U^{t} \rightarrow[0, \infty)$ by

$$
A\left(u_{1}, u_{2}, \ldots, u_{t-1}, u_{t}\right)=\sum_{i=1}^{t} \sum_{i<j}\left|u_{i}-u_{j}\right|
$$

for all $u_{i} \in U, i=1,2, \ldots, t$. Then $(U, A)$ is complete $A$-metric space. 
If we define $T: U \rightarrow U$ by $T u=\frac{2 u}{7}$, then $T$ satisfy the conditions of Theorem 2.3. For all $u_{i} \in U, i=1,2, \ldots, t$,

$$
\begin{aligned}
A\left(T u_{1}, T u_{2}, \ldots, T u_{t-1}, T u_{t}\right)= & A\left(\frac{2 u_{1}}{7}, \frac{2 u_{2}}{7}, \ldots, \frac{2 u_{t-1}}{7}, \frac{2 u_{t}}{7}\right) \\
= & \frac{2}{7}\left|u_{1}-u_{2}\right|+\frac{2}{7}\left|u_{1}-u_{3}\right|+\ldots+\frac{2}{7}\left|u_{1}-u_{t}\right| \\
& +\frac{2}{7}\left|u_{2}-u_{3}\right|+\frac{2}{7}\left|u_{2}-u_{4}\right|+\ldots+\frac{2}{7}\left|u_{2}-u_{t}\right| \\
& \vdots \\
& +\frac{2}{7}\left|u_{t-2}-u_{t-1}\right|+\frac{2}{7}\left|u_{t-2}-u_{t}\right|+\frac{2}{7}\left|u_{t-1}-u_{t}\right| \\
= & \frac{2}{7} \sum_{i=1}^{t} \sum_{i<j}\left|u_{i}-u_{j}\right| \\
= & \frac{2}{7} A\left(u_{1}, u_{2}, \ldots, u_{t-1}, u_{t}\right)
\end{aligned}
$$

where $\frac{2}{7} \in[0,1)$. This implies that $T$ is a $A Z$ mapping. And $u=0$ is the unique fixed point of $T$ in $U$ as asserted by Theorem 2.3.

\section{CONFLiCTS OF INTEREST}

The author declares that there are no conflicts of interest regarding the publication of this article.

\section{REFERENCES}

[1] Abbas, M., Ali, B., Suleiman Y.I.,Generalized coupled common fixed point results in partially ordered A-metric spaces, Fixed Point Theory Appl., Article Number: 64(2015).

[2] Banach, S., Sur les operations dans les ensembles abstraits et leur application aux equations integrals, Fund. Math., 2(1922), $133-181$.

[3] Chatterjea, S.K., Fixed point theorems, C. R. Acad. Bulgare Sci., 25(6)(1972), 727-730.

[4] Fernandez, J., Saelee, S., Saxena, K., Malviya, N., Kumam, P., The A-cone metric space over Banach algebra with applications, Cogent Math., 4(1)(2017).

[5] Kannan, R., Some results on fixed points, Bull. Calc. Math. Soc., 60(1)(1968), 71-77.

[6] Mustafa, Z., Sims, B., A new approach to generalized metric spaces, J. Nonlinear Convex Anal., 7(2)(2006), 289-297.

[7] Sedghi, S., Shobe, N., Aliouche, A., A generalization of fixed point theorems in S-metric spaces, Mat. Vesn., 64(3)(2012), $258-266$.

[8] Sedghi, S., Shobe, N, Zhou, H., A common fixed point theorem in $D^{*}$-metric spaces, Fixed Point Theory Appl., Article Number:27906(2007).

[9] Zamfirescu, T., Fix point theorems in metric spaces, Arch. Math., 23(1972), 292-298. 